\title{
KdV Equation in the Quarter-Plane: Evolution of the Weyl Functions and Unbounded Solutions
}

\author{
A. Sakhnovich* \\ Department of Mathematics, University of Vienna, Nordbergstrasse 15, A-1090 Vienna, Austria
}

\begin{abstract}
The matrix KdV equation with a negative dispersion term is considered in the right upper quarter-plane. The evolution law is derived for the Weyl function of a corresponding auxiliary linear system. Using the low energy asymptotics of the Weyl functions, the unboundedness of solutions is obtained for some classes of the initial-boundary conditions.
\end{abstract}

Keywords and phrases: KdV, initial-boundary value problem, Weyl function, evolution, low-energy asymptotics, blow-up solution

Mathematics Subject Classification: 35Q53, 34B20, 35G31

\section{Introduction}

Initial-boundary value problems for the integrable nonlinear equations are of great interest, see, for instance, $[1,4,9,10,15,16,22,24,26,27]$ and references therein. In particular, many interesting works were dedicated to the scalar Korteweg-de Vries (KdV) equation $u_{t}+6 u u_{x} \pm u_{x x x}=0$ in the quarterplane $x \geq 0, t \geq 0$. Solitons induced by boundary excitation were first investigated numerically in $[2,5]$. Some rigorous sufficient conditions for the existence of the unique global $\mathrm{KdV}$ solution in a semistrip were given in [28], where the case that $u(0, t)=0$ was studied (and the requirement $u_{x}(0, t)=0$ was added when the sign in front of the dispersion term $u_{x x x}$ was negative). The restriction $u(0, t)=0$ on the boundary condition (for the case of the "plus" in front of the dispersion term in KdV) was removed in the important paper [3]. The so called global relation approach, introduced by A.S. Fokas, was applied to KdV in [29] (see also references therein) and an integral representation for the solution was derived by inverting this global relation. However, after getting the integral representation, "one is left with a nonlinear integral equation to solve" (see [1]). Scattering problems (so called elbow scattering) for linear systems, which are associated with a scalar KdV (i.e., for Lax and generalized Lax systems), and some aspects of the initialboundary value problem for KdV in the quarterplane were treated in a series of papers by P.C. Sabatier [18-20]. In spite of interesting results, some difficult problems remain unsolved there as well, which is shortly stated in the summary of [19]: "if the approach is transposed to the quarterplane problem, it shows a generalization to KdV of the solutions obtained by Fokas in the linearized KdV problem, but unfortunately the last step is iterative and complicated". Thus, the initial-boundary value problem for $\mathrm{KdV}$ is interesting, actively studied and quite difficult even in the scalar case. In this paper we introduce

*Corresponding author. E-mail: Oleksandr.Sakhnovych@univie.ac.at 
Weyl theory and, in particular, the evolution of the Weyl function for the matrix KdV equation with the minus sign in front of the dispersion term $u_{x x x}$ :

$$
u_{t}+3\left(u u_{x}+u_{x} u\right)-u_{x x x}=0
$$

where $u(x, t)$ is an $m \times m$ matrix function and $\mathrm{KdV}$ is considered in the quarterplane. We study in detail an interesting particular case and construct also blow-up solutions.

Equation (1.1) is the compatibility condition of the auxiliary linear systems

$$
\begin{gathered}
\Phi_{x}(x, t, z)=G(x, t, z) \Phi(x, t, z), \\
\Phi_{t}(x, t, z)=F(x, t, z) \Phi(x, t, z), \\
G:=\left[\begin{array}{cc}
0 & I_{m} \\
u-z I_{m} & 0
\end{array}\right], \\
F:=\left[\begin{array}{cc}
u_{x} \\
u_{x x}-2\left(u+2 z I_{m}\right)\left(u-z I_{m}\right) & -2\left(u+2 z I_{m}\right) \\
-u_{x}
\end{array}\right],
\end{gathered}
$$

where $I_{m}$ is the $m \times m$ identity matrix. In other words equation (1.1) is equivalent to the zero curvature equation

$$
G_{t}-F_{x}+[G, F]=0, \quad[G, F]:=G F-F G,
$$

where $G$ and $F$ are given by (1.4) and (1.5), respectively.

System (1.2), (1.4) is equivalent to the canonical system (2.10) (and to the Schrödinger equation), and in this paper we derive the evolution $M(t, z)$ of the Weyl function of this system. This evolution is an important component of the solution of the initial-boundary value problem. For simplicity, we derive the evolution under condition that $F$ and $G$ are continuously differentiable, though the requirement of the continuous differentiability could be weakened using the results from [23].

If $u(0, t)=u_{x x}(0, t)=0$, then system (1.3) at $x=0$ is equivalent to a Dirac system and its Weyl function is expressed via $M(0, z)$ (see formula (3.21)). We apply (3.21) and low energy asymptotics of $M(0, z)$ to show the unboundedness of the KdV solutions in the quarter-plane for some classes of simple initial conditions $u(x, 0)$.

Our Weyl function $M(t, z)$ is connected with the Weyl function from [6] (the latter being denoted here by $\mathcal{M}(t, z))$ via the linear fractional transformation $M=\left(\mathcal{M}-I_{m}\right)\left(\mathcal{M}+I_{m}\right)^{-1}$. We note that the high energy asymptotics of the Weyl functions was actively studied (see $[6,7,17,21]$ and references therein) following the seminal papers $[12,13]$. Though the low energy asymptotics of the Weyl functions is used in the present paper, the high energy asymptotics (namely, an important result on asymptotics of the Weyl function in terms of the values of $u$ and its derivatives at $x=0$ from [6]) jointly with the evolution of the Weyl function could also prove useful for the analysis of the initial-boundary conditions.

We discuss some background in Section 2, obtain the evolution law in Section 3, and study the unboundedness of the solutions in Section 4.

\section{Some Background}

Let us normalize the fundamental solution $\Psi$ of the equation (1.2) by introducing

$$
\Psi(x, t, z)=\Phi(x, t, z) \Phi(0, t, z)^{-1}
$$

satisfying the initial condition

$$
\Psi(0, t, z)=I_{2 m} .
$$


Suppose, $G$ and $F$ are continuously differentiable on the half-strip $0 \leq x<\infty, 0 \leq t<\mathbf{t} \leq \infty$ and (1.6) holds. Then, according to section $12.1[27]$ (see also $[25,26]$ ) we have

$$
\Psi(x, t, z)=V(x, t, z) \Psi(x, 0, z) V(0, t, z)^{-1},
$$

where the $2 m \times 2 m$ matrix function $V$ satisfies relations

$$
V_{t}(x, t, z)=F(x, t, z) V(x, t, z), \quad V(x, 0, z)=I_{2 m}
$$

Introduce the matrices

$$
\begin{gathered}
J:=\left[\begin{array}{cc}
0 & I_{m} \\
I_{m} & 0
\end{array}\right], \quad \Sigma_{3}:=\left[\begin{array}{cc}
I_{m} & 0 \\
0 & -I_{m}
\end{array}\right], \\
J_{1}=T J T^{*}=i\left[\begin{array}{cc}
0 & -I_{m} \\
I_{m} & 0
\end{array}\right], \quad T:=\frac{1}{\sqrt{2}}\left[\begin{array}{cc}
i I_{m} & I_{m} \\
i I_{m} & -I_{m}
\end{array}\right] .
\end{gathered}
$$

Further we shall consider the case of the self-adjoint (real-valued for $m=1$ ) $u$ :

$$
u(x, t)=u(x, t)^{*}, \text { i.e., } \frac{\partial}{\partial x}\left(\Psi(x, t, 0)^{*} J_{1} \Psi(x, t, 0)\right)=0 .
$$

From (2.2), (2.6) and (2.7) it follows that

$$
T^{*} \Psi(x, t, 0)^{*} J_{1} \Psi(x, t, 0) T=T^{*} J_{1} T=J .
$$

Putting

$$
\widetilde{\Psi}(x, t, z)=(\Psi(x, t, 0) T)^{-1} \Psi(x, t, z) T,
$$

and taking into account $(1.2),(1.4),(2.8)$ and $(2.9)$ we see that $\widetilde{\Psi}(x, t, z)$ is the fundamental solution of the canonical system

$$
\widetilde{\Psi}_{x}(x, t, z)=i z J H(x, t) \widetilde{\Psi}(x, t, z), \quad \widetilde{\Psi}(0, t, z)=I_{2 m},
$$

where

$$
H(x, t)=T^{*} \Psi(x, t, 0)^{*}\left[\begin{array}{cc}
I_{m} & 0 \\
0 & 0
\end{array}\right] \Psi(x, t, 0) T \geq 0 .
$$

Moreover, $H$ satisfies [25] the positivity condition

$$
\int_{0}^{l} H(s, t) d s>0 \quad(l>0) .
$$

Indeed, for any $h \in \mathbb{C}^{2 m}, h \neq 0$ we have,

$$
h^{*} H(s, t) h=g(s, t)^{*} g(s, t), \quad g(s, t):=\left[\begin{array}{ll}
I_{m} & 0
\end{array}\right] \Psi(s, t, 0) T h,
$$

where, according to (1.2), (1.4), and (2.2), the relations

$$
g_{s s}(s, t)=u(s, t) g(s, t), \quad\left[\begin{array}{c}
g(0, t) \\
g_{s}(0, t)
\end{array}\right]=T h \neq 0
$$

hold. Inequality (2.12) follows from (2.13) and (2.14) .

By (2.12), the linear fractional transformations

$$
\begin{aligned}
M(l, t, z)= & i\left(\mathcal{A}_{11}(l, t, z) P_{l}(t, z)+\mathcal{A}_{12}(l, t, z) Q_{l}(t, z)\right) \\
& \times\left(\mathcal{A}_{21}(l, t, z) P_{l}(t, z)+\mathcal{A}_{22}(l, t, z) Q_{l}(t, z)\right)^{-1}, \quad \Im(z)>0
\end{aligned}
$$


where the matrices $\mathcal{A}_{k j}$ are the $m \times m$ blocks of $\mathcal{A}$,

$$
\mathcal{A}(l, t, z):=\widetilde{\Psi}(l, t, \bar{z})^{*},
$$

and $P_{l}, Q_{l}$ are meromorphic nonsingular pairs with property- $J$,

$$
P_{l}^{*} P_{l}+Q_{l}^{*} Q_{l}>0, \quad P_{l}^{*} Q_{l}+Q_{l}^{*} P_{l} \geq 0,
$$

are well-defined for $\Im(z)>0$. The matrix functions $M$ are Herglotz (Nevanlinna) functions, that is, $\Im(M(z)) \geq 0$ in $\mathbb{C}_{+}$, and they are called Weyl-functions of the canonical system on the interval $(0, l)$. Further we shall assume that $u$ is bounded:

$$
\sup _{0 \leq x<\infty, 0 \leq t<\mathbf{t}}\|u(x, t)\|<C .
$$

Then, by (2.10) and (2.12) there is a unique limit of the functions $M(l, t, z)$, which is independent of the choice of the pairs $P_{l}, Q_{l}$ with property- $J$ :

$$
\lim _{l \rightarrow \infty} M(l, t, z)=M(t, z) .
$$

Fore a detailed proof of (2.19) see p. 177 in [27], where the proof of a similar formula (1.18) (condition b)) from p. 169 is given.

Note that one can omit the variable $t$ in formulas (1.2), (2.1), (2.2), (2.9)-(2.19) while considering a certain subclass of canonical systems. The limit $M(z)=\lim _{l \rightarrow \infty} M(l, z)$ is called the Weyl-function of the system (2.10) on the semi-axis $x>0$. It has the property (see formula (1.24) on p. 121 in [27])

$$
\int_{0}^{\infty}\left[I_{m} i M(z)^{*}\right] \widetilde{\Psi}(x, z)^{*} H(x) \widetilde{\Psi}(x, z)\left[\begin{array}{c}
I_{m} \\
-i M(z)
\end{array}\right] d x<\infty, \quad z \in \mathbb{C}_{+} .
$$

The function $M(z)$ is also the Weyl-function of the Sturm-Liouville system

$$
-Y_{x x}(x, z)+u(x) Y(x, z)=z Y(x, z),
$$

where the matrix function $u$ coincides with the $u$ in (1.4). In particular, formula (2.20) can be rewritten in the form

$$
\int_{0}^{\infty}\left[I_{m} i M(z)^{*}\right] Y(x, z)^{*} Y(x, z)\left[\begin{array}{c}
I_{m} \\
-i M(z)
\end{array}\right] d x<\infty, \quad z \in \mathbb{C}_{+},
$$

where $Y$ is the $m \times 2 m$ solution of (2.21) normalized by the condition

$$
Y(0, z)=(\sqrt{2})^{-1}\left[\begin{array}{ll}
i I_{m} & I_{m}
\end{array}\right], \quad Y_{x}(0, z)=(\sqrt{2})^{-1}\left[\begin{array}{ll}
i I_{m} & -I_{m}
\end{array}\right] .
$$

We also recall that the Weyl-function $M_{D}(\zeta)$ of the Dirac-type system on the semi-axis

$$
\frac{d}{d t} W(t, \zeta)=i\left[\zeta \Sigma_{3}+\Sigma_{3} \mathcal{V}(t)\right] W(t, \zeta), \quad W(0, \zeta)=I_{2 m}, \quad \mathcal{V}=\left[\begin{array}{cc}
0 & v \\
v^{*} & 0
\end{array}\right]
$$

where $\mathcal{V}$ is locally summable, is uniquely defined by the inequality

$$
\begin{aligned}
& \int_{0}^{\infty}\left[I_{m} \quad i M_{D}(\zeta)^{*}\right] K W(t, \zeta)^{*} W(t, \zeta) K^{*}\left[\begin{array}{c}
I_{m} \\
-i M_{D}(\zeta)
\end{array}\right] d t<\infty, \\
& \Im(\zeta)>0, \quad K:=\frac{1}{\sqrt{2}}\left[\begin{array}{cc}
I_{m} & -I_{m} \\
I_{m} & I_{m}
\end{array}\right] .
\end{aligned}
$$

See the procedure to recover $\mathcal{V}$ from $M_{D}$ in $[21,27]$ and the references therein.

Using (2.3) and (2.19) the evolution of the Weyl-function $M(t, z)(t>0)$ was derived in [25] -[27] for the KdV equation $u_{t}-3\left(u u_{x}+u_{x} u\right)+u_{x x x}=0$ with the plus sign in front of the dispersion term. Moreover, the initial-boundary problem $u(x, 0)=f(x), u(0, t)=u_{x x}(0, t)=0$ was treated in [25] for the scalar case $u_{t}-6 u u_{x}+u_{x x x}=0$. We shall modify these results for the case of the KdV equation (1.1), where this number of the initial-boundary conditions will be appropriate (see $[3,28])$. 


\section{The KdV Equation with a Negative Dispersion Term}

Denote the Weyl-function of system $(2.10)$ at $t=0$ by $M(0, z)$ and put

$$
\begin{aligned}
& R(l, t, z):=(\Psi(l, t, 0) T)^{*}\left(V(l, t, \bar{z})^{*}\right)^{-1}\left((\Psi(l, 0,0) T)^{*}\right)^{-1}, \\
& R(t, z)=\left[\begin{array}{ll}
r_{11}(t, z) & r_{12}(t, z) \\
r_{21}(t, z) & r_{22}(t, z)
\end{array}\right]:=R(0, t, z),
\end{aligned}
$$

where $r_{k j}$ are $m \times m$ blocks of $R$.

Proposition 3.1. Let the bounded $m \times m$ matrix function u satisfy the KdV equation (1.1) on the halfstrip $0 \leq x<\infty, 0 \leq t<\mathbf{t} \leq \infty$. Assume that the corresponding matrix functions $G$ and $F$ given by (1.4) and (1.5) are continuously differentiable. Then the evolution of the Weyl-function $M(t, z)$ is given by the formula

$$
M(t, z)=i\left((-i) r_{11}(t, z) M(0, z)+r_{12}(t, z)\right)\left((-i) r_{21}(t, z) M(0, z)+r_{22}(t, z)\right)^{-1} .
$$

Proof. Taking into account (2.9) and (2.16), rewrite formula (2.3) in the form

$$
\mathcal{A}(l, t, z) R(l, t, z)=R(t, z) \mathcal{A}(l, 0, z) .
$$

To show that $R$ is $J$-expanding in some domain in $\mathbb{C}_{+}$, we shall use the equation

$$
\frac{\partial}{\partial t}\left(V(l, t, \bar{z})^{-1}\right)=-V(l, t, \bar{z})^{-1} F(l, t, \bar{z})
$$

From (3.5) it follows that

$$
\begin{aligned}
& \frac{\partial}{\partial t}\left(V(l, t, \bar{z})^{-1} J_{1}\left(V(l, t, \bar{z})^{-1}\right)^{*}\right) \\
& =-V(l, t, \bar{z})^{-1}\left(F(l, t, \bar{z}) J_{1}+J_{1} F(l, t, \bar{z})^{*}\right)\left(V(l, t, \bar{z})^{-1}\right)^{*} .
\end{aligned}
$$

By (1.5) and the first relation in (2.7) we have

$$
F(l, t, \bar{z}) J_{1}+J_{1} F(l, t, \bar{z})^{*}=2 i(z-\bar{z})\left[\begin{array}{cc}
2 I_{m} & 0 \\
0 & 2(z+\bar{z}) I_{m}-u(l, t)
\end{array}\right] .
$$

Taking into account (2.18) and (3.7) we derive

$$
-\left(F(l, t, \bar{z}) J_{1}+J_{1} F(l, t, \bar{z})^{*}\right)>0 \quad \text { for } \quad \Im(z)>0, \Re z>C / 4 .
$$

In view of (3.6), (3.8) and the second relation in (2.4) we get

$$
V(l, t, \bar{z})^{-1} J_{1}\left(V(l, t, \bar{z})^{-1}\right)^{*}>J_{1} \quad \text { for } \quad \Im(z)>0, \Re z>C / 4 .
$$

According to (2.8), (3.1) and (3.9) the inequality

$$
R(l, t, z)^{*} J R(l, t, z)>J \quad \text { for } \quad \Im(z)>0, \Re z>C / 4
$$

is true. By (2.15), (2.19), (3.4) and (3.10), we derive (3.3) for $z$ in the domain $\Im(z)>0, \Re z>C / 4$. In view of the analyticity of the Weyl-functions, it follows that (3.3) is valid everywhere in $\mathbb{C}_{+}$.

Consider now the particular case of the initial-boundary value problem in the quarter-plane:

$$
u(x, 0)=f(x), \quad u(0, t)=u_{x x}(0, t)=0 \quad(0 \leq x<\infty, 0 \leq t<\infty) .
$$


If $u$ is a scalar function, the case (3.11) belongs to the so called "linearizable" cases from [8, Section 4.3] but we shall see that, in spite of being "linearizable", this case is not so simple. In particular, the blow up solutions, that appear here, are of interest. As already mentioned above, the KdV equation, which has the "plus" in front of the dispersion term, was treated together with conditions (3.11) in [26] but these conditions seem more appropriate in the "minus" case. We recall also the somewhat different conditions of this type (namely, $\left.u(0, t)=u_{x}(0, t)=0\right)$ in [28].

According to (2.2), (3.1), (3.2) and (3.5) we have

$$
\begin{aligned}
& R(t, z)=T^{*}\left(V(0, t, \bar{z})^{*}\right)^{-1} T \\
& \frac{d}{d t} R(t, z)=-T^{*} F(0, t, \bar{z})^{*} T R(t, z), \quad R(0, z)=I_{2 m} .
\end{aligned}
$$

By (1.5) and (3.11) one can see that

$$
-F(0, t, \bar{z})^{*}=\left[\begin{array}{cc}
-u_{x}(0, t) & -4 z^{2} I_{m} \\
4 z I_{m} & u_{x}(0, t)
\end{array}\right] .
$$

Following [25], let us transform (3.13) into the Dirac-type system. Note for that purpose, that

$$
\begin{gathered}
T \operatorname{diag}\left\{I_{m}, \sqrt{z} I_{m}\right\}\left[\begin{array}{cc}
0 & -z^{2} I_{m} \\
z I_{m} & 0
\end{array}\right] \operatorname{diag}\left\{I_{m}, \frac{1}{\sqrt{z}} I_{m}\right\} T^{*}=-i z^{\frac{3}{2}} \Sigma_{3}, \\
T \operatorname{diag}\left\{I_{m}, \sqrt{z} I_{m}\right\} \Sigma_{3} \operatorname{diag}\left\{I_{m}, \frac{1}{\sqrt{z}} I_{m}\right\} T^{*}=J,
\end{gathered}
$$

where $J$ and $j$ are defined in $(2.5)$ and diag means a block diagonal matrix. We consider $z \in \mathbb{C}_{+}$and choose the branch $\sqrt{z}$ so that $\sqrt{z} \in \mathbb{C}_{+}$. Now, put

$$
\begin{aligned}
& \widetilde{R}(t, \zeta):=Z(z)^{-1} R(t, z) Z(z), \quad Z(z):=T^{*} \operatorname{diag}\left\{I_{m}, \frac{1}{\sqrt{z}} I_{m}\right\} T^{*}, \\
& \zeta:=-4 z^{\frac{3}{2}} .
\end{aligned}
$$

From (3.13)-(3.18) it follows that $\widetilde{R}$ satisfies the Dirac-type system

$$
\frac{d}{d t} \widetilde{R}(t, \zeta)=\left[i \zeta \Sigma_{3}-\operatorname{diag}\left\{u_{x}(0, t), u_{x}(0, t)\right\} J\right] \widetilde{R}(t, \zeta), \quad \widetilde{R}(0, \zeta)=I_{2 m}
$$

Recall that the Weyl-function $M_{D}$ of the Dirac-type system is defined via (2.25). Recall also that the Weyl-function $M_{t r}$ of the Sturm-Liouville system with the trivial potential $u$ (i.e., $u$ equal to zero) equals $[i \sqrt{z}-1] /[i \sqrt{z}+1] I_{m}$. Hence we shall require that

$$
\lim _{t \rightarrow \infty} M(t, z)=\frac{i \sqrt{z}-1}{i \sqrt{z}+1} I_{m}
$$

Proposition 3.2. Assume that there exists a solution $u$ of the KdV equation (1.1) on the quarter-plane $0 \leq x<\infty, 0 \leq t<\infty$, which satisfies also the conditions of Proposition 3.1 and the initial-boundary value conditions (3.11). Suppose that (3.20) holds. Then u may be uniquely recovered by the following procedure:

First, the Weyl-function of the Dirac-type system (3.19) is recovered for sufficiently large values of $\Im(\sqrt{z})$ by the formula

$$
M_{D}\left(-4 z^{\frac{3}{2}}\right)=\frac{1}{\sqrt{z}}\left(I_{m}+M(0, z)\right)\left(I_{m}-M(0, z)\right)^{-1},
$$

where $z$ belongs to the sector $\frac{2}{3} \pi<\arg (z)<\pi$. The matrix function $M(0, z)$ in (3.21) is the Weylfunction of the canonical system (2.10), (2.11) at $t=0$, which is determined by the initial condition $u(x, 0)=f(x)$. 
Next, the matrix-function $u_{x}(0, t)$ is uniquely recovered from $M_{D}(z)$, after which $R(t, z)$ is given by (3.13) and (3.14). The evolution of the Weyl-function $M(t, z)$ is given by (3.3) in terms of $R$ and $M(0, z)$.

Finally, $u(x, t)$ is uniquely recovered from $M(t, z)$.

Proof. By (3.19) we get

$$
\frac{d}{d t} \widetilde{R}(t, \zeta)^{*} \Sigma_{3} \widetilde{R}(t, \zeta)=i(\zeta-\bar{\zeta}) \widetilde{R}(t, \zeta)^{*} \widetilde{R}(t, \zeta) .
$$

Formula (3.22) and the second relation in (3.19) imply that

$$
\widetilde{R}(t, \zeta)^{*} \Sigma_{3} \widetilde{R}(t, \zeta)-\Sigma_{3}<-\delta \int_{0}^{t} \widetilde{R}(s, \zeta)^{*} \widetilde{R}(s, \zeta) d s \quad \text { for } \Im(\zeta)>\delta / 2>0,
$$

or, equivalently, we have

$$
\Sigma_{3}-\widetilde{R}(t, \zeta)^{*} \Sigma_{3} \widetilde{R}(t, \zeta)>\delta \int_{0}^{t} \widetilde{R}(s, \zeta)^{*} \widetilde{R}(s, \zeta) d s \quad \text { for } \Im(\zeta)>\delta / 2>0 .
$$

Next, let us show that for sufficiently large values of $\Im(\sqrt{z})$ and $t$ the inequality

$$
\left[I_{m} \quad i M_{D}(\zeta)^{*}\right] K \widetilde{R}(t, \zeta)^{*} \Sigma_{3} \widetilde{R}(t, \zeta) K^{*}\left[\begin{array}{c}
I_{m} \\
-i M_{D}(\zeta)
\end{array}\right] \geq 0
$$

where $M_{D}$ is given by (3.21), is valid. First, take into account (2.26) and (3.17) and note that

$$
Z(z) K^{*}=-\frac{1}{\sqrt{2 z}}\left[\begin{array}{cc}
i I_{m} & \sqrt{z} I_{m} \\
I_{m} & i \sqrt{z} I_{m}
\end{array}\right]
$$

Using (3.17), (3.21) and (3.25) we write

$$
\widetilde{R}(t, \zeta) K^{*}\left[\begin{array}{c}
I_{m} \\
-i M_{D}(\zeta)
\end{array}\right]=\frac{-2}{\sqrt{2 z}} Z(z)^{-1} R(t, z)\left[\begin{array}{c}
-i M(0, z) \\
I_{m}
\end{array}\right]\left(I_{m}-M(0, z)\right)^{-1} .
$$

According to Proposition 3.1 we have

$$
R(t, z)\left[\begin{array}{c}
-i M(0, z) \\
I_{m}
\end{array}\right]=\left[\begin{array}{c}
-i M(t, z) \\
I_{m}
\end{array}\right]\left((-i) r_{21}(t, z) M(0, z)+r_{22}(t, z)\right) .
$$

Taking into account that

$$
Z(z)^{-1}=T \operatorname{diag}\left\{I_{m}, \sqrt{z} I_{m}\right\} T, \quad T^{*} \Sigma_{3} T=J_{1}
$$

we obtain

$$
\left(Z(z)^{-1}\right)^{*} \Sigma_{3} Z(z)^{-1}=\frac{1}{2}\left[\begin{array}{cc}
i(\overline{\sqrt{z}}-\sqrt{z}) I_{m} & (\overline{\sqrt{z}}+\sqrt{z}) I_{m} \\
(\overline{\sqrt{z}}+\sqrt{z}) I_{m} & i(\sqrt{z}-\sqrt{z}) I_{m}
\end{array}\right] .
$$

From (3.26), (3.27) and (3.29) it follows that

$$
\begin{aligned}
& {\left[I_{m} \quad i M_{D}(\zeta)^{*}\right] K \widetilde{R}(t, \zeta)^{*} \Sigma_{3} \widetilde{R}(t, \zeta) K^{*}\left[\begin{array}{c}
I_{m} \\
-i M_{D}(\zeta)
\end{array}\right]} \\
& =\omega(t, z)^{*}\left[i M(t, z)^{*} \quad I_{m}\right]\left[\begin{array}{cc}
i(\overline{\sqrt{z}}-\sqrt{z}) I_{m} & (\overline{\sqrt{z}}+\sqrt{z}) I_{m} \\
(\overline{\sqrt{z}}+\sqrt{z}) I_{m} & i(\sqrt{z}-\sqrt{z}) I_{m}
\end{array}\right] \\
& \times\left[\begin{array}{c}
-i M(t, z) \\
I_{m}
\end{array}\right] \omega(t, z) \\
& \sim \frac{8|\sqrt{z}|^{2}}{|i \sqrt{z}+1|^{2}} \omega(t, z)^{*} \omega(t, z)>0 \quad(t \rightarrow \infty),
\end{aligned}
$$


where

$$
\omega(t, z)=\frac{1}{\sqrt{z}}\left((-i) r_{21}(t, z) M(0, z)+r_{22}(t, z)\right)\left(I_{m}-M(0, z)\right)^{-1} .
$$

We recall the choice $\frac{2}{3} \pi<\arg (z)<\pi$, that is, $\Im(\zeta)>0$. By (3.20) and (3.30) for sufficiently large values of $t$ we get (3.24).

Hence, it follows from (3.23) and (3.24) that the inequality

$$
\int_{0}^{\infty}\left[I_{m} \quad i M_{D}(\zeta)^{*}\right] K \widetilde{R}(s, \zeta)^{*} \widetilde{R}(s, \zeta) K^{*}\left[\begin{array}{c}
I_{m} \\
-i M_{D}(\zeta)
\end{array}\right] d s<\infty
$$

holds. Thus, $M_{D}$ is, indeed, the Weyl-function of the Dirac system. The evolution $M(t, z)$ follows from Proposition 3.1. For the inverse problem for our canonical system, when $u$ is bounded, see [27], p. 116 and references.

We provide a short SUMMARY of the scheme employed:

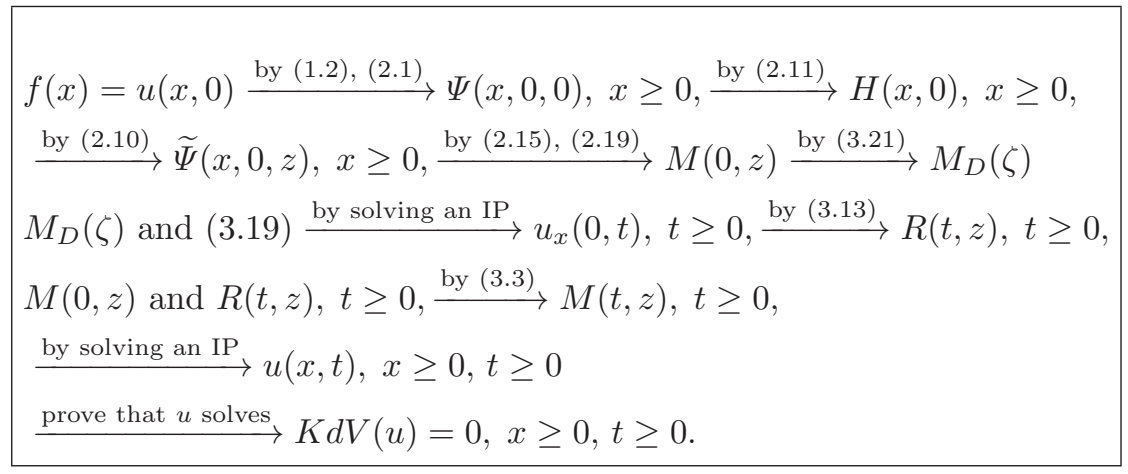

Consider the simplest example.

Example 3.3. Put for simplicity $m=1$, i.e., consider a scalar $K d V$ equation. The simplest case is the case $u(x, 0)=f(x)=0$ (see the initial-boundary value conditions $(3.11)$ ). The Weyl function $M(0, z)$ of the Sturm-Liouville system with $u \equiv 0$ is given by the formula

$$
M(0, z)=\frac{i \sqrt{z}-1}{i \sqrt{z}+1} .
$$

By (3.21) it follows that the Weyl function $M_{D}(\zeta)$ of the Dirac system (3.19) is given by the formula

$$
M_{D}(\zeta(z))=\frac{1}{\sqrt{z}} \frac{1+\frac{i \sqrt{z}-1}{i \sqrt{z}+1}}{1-\frac{i \sqrt{z}-1}{i \sqrt{z}+1}}=i, \quad \zeta(z)=-4 z^{\frac{3}{2}} .
$$

As $M_{D} \equiv i$ is the Weyl function of the Dirac system (3.19) with a trivial potential $u_{x}=0$ we get the fundamental solution

$$
\widetilde{R}(t, \zeta)=\exp \left(i t \zeta \Sigma_{3}\right)
$$

Hence, taking into account (3.17) we derive

$$
R(t, \zeta(z))=Z(z) \exp \left(i t \zeta(z) \Sigma_{3}\right) Z(z)^{-1}, \quad Z(z):=T^{*} \operatorname{diag}\left\{I_{m}, \frac{1}{\sqrt{z}} I_{m}\right\} T^{*} .
$$

Using (3.36) we can obtain $M(t, z)$. First, rewrite (3.3) in the form

$$
\begin{aligned}
M(t, z)= & i\left[\begin{array}{ll}
1 & 0
\end{array}\right] R(t, \zeta(z)) \\
& \times\left[\begin{array}{c}
-i M(0, z) \\
1
\end{array}\right]\left(\left[\begin{array}{ll}
0 & 1
\end{array}\right] R(t, \zeta(z))\left[\begin{array}{c}
-i M(0, z) \\
1
\end{array}\right]\right)^{-1} .
\end{aligned}
$$


Next, note that according to (3.33) and the second equality in (3.36) we have

$$
Z(z)^{-1}\left[\begin{array}{c}
-i M(0, z) \\
1
\end{array}\right]=-\frac{2 \sqrt{z}}{i \sqrt{z}+1}\left[\begin{array}{l}
1 \\
0
\end{array}\right]
$$

From (3.36) and (3.38) it follows that

$$
R(t, \zeta(z))\left[\begin{array}{c}
-i M(0, z) \\
1
\end{array}\right]=\frac{e^{i t \zeta(z)}}{i \sqrt{z}+1}\left[\begin{array}{c}
\sqrt{z}+i \\
i \sqrt{z}+1
\end{array}\right]=e^{i t \zeta(z)}\left[\begin{array}{c}
-i M(0, z) \\
1
\end{array}\right] .
$$

By (3.37) and (3.39) we have $M(t, z) \equiv M(0, z)$. That is, $u(x, t) \equiv 0$.

\section{Non-existence of the global solutions in the quarter-plane}

The global solutions, satisfying conditions of Proposition 3.2, do not exist for wide classes of the initial value functions $f(x)$. Using small energy asymptotics of the corresponding Weyl-functions we explicitly construct in this section such a class of initial value functions.

First, we describe the explicit construction of the potentials and Weyl functions from Theorem 0.1 and Proposition 2.2 in [14]. For this purpose we fix an integer $n>0$ and three matrices, namely, an $n \times n$ matrix $\alpha$ and $n \times m$ matrices $\vartheta_{k}, k=1,2$, such that

$$
\alpha-\alpha^{*}=\vartheta_{1} \vartheta_{2}^{*}-\vartheta_{2} \vartheta_{1}^{*} .
$$

The triple $\left\{\alpha, \vartheta_{1}, \vartheta_{2}\right\}$, which satisfies (4.1), is called admissible. Consider Sturm-Liouville system (2.21) where $u$ is determined by the triple $\left\{\alpha, \vartheta_{1}, \vartheta_{2}\right\}$. Namely, put

$$
\begin{aligned}
u(x)= & 2\left\{\left(\Lambda_{2}(x)^{*} S(x)^{-1} \Lambda_{2}(x)\right)^{2}+\Lambda_{1}(x)^{*} S(x)^{-1} \Lambda_{2}(x)\right. \\
& \left.+\Lambda_{2}(x)^{*} S(x)^{-1} \Lambda_{1}(x)\right\},
\end{aligned}
$$

where

$$
\begin{gathered}
\Lambda(x)=\left[\begin{array}{l}
\Lambda_{1}(x) \\
\Lambda_{2}(x)
\end{array}\right]=e^{x \beta}\left[\begin{array}{l}
\vartheta_{1} \\
\vartheta_{2}
\end{array}\right], \quad \beta=\left[\begin{array}{ll}
0 & \alpha \\
-I_{n} & 0
\end{array}\right], \\
S(x)=I_{n}+\int_{0}^{x} \Lambda_{2}(y) \Lambda_{2}(y)^{*} d y, \quad x \geq 0 .
\end{gathered}
$$

Theorem 4.1. [14] Let $u$ be determined by the admissible triple $\left\{\alpha, \vartheta_{1}, \vartheta_{2}\right\}$ via formulas (4.2)-(4.5) and let $Y$ satisfy (2.21) and (2.23). Then, for any sufficiently large values of $\Im \sqrt{z}\left(z, \sqrt{z} \in \mathbb{C}_{+}\right)$we have

$$
\int_{0}^{\infty}\left[i \phi(\sqrt{z})^{*} I_{m}\right] Y(x, z)^{*} Y(x, z)\left[\begin{array}{c}
-i \phi(\sqrt{z}) \\
I_{m}
\end{array}\right] d x<\infty
$$

where

$$
\phi(\sqrt{z})=\left(\varphi_{2}(z)+\frac{2 i}{\sqrt{z}} I_{m}\right) \varphi_{1}(z),
$$

and the matrix functions $\varphi_{1}$ and $\varphi_{2}$ are rational matrix functions given by the realizations:

$$
\varphi_{1}(z)^{-1}=I_{m}+B^{*} \hat{J}\left(z I_{2 n+m}-A\right)^{-1} B, \quad \varphi_{2}(z)=-I_{m}+C\left(z I_{2 n+m}-A\right)^{-1} B,
$$




$$
\begin{aligned}
& A=\left[\begin{array}{ccc}
\alpha^{*} & 0 & 0 \\
\vartheta_{1}^{*} & 0 & 0 \\
\vartheta_{2} \vartheta_{2}^{*} & \vartheta_{1} & \alpha
\end{array}\right], \quad B=\left[\begin{array}{c}
\vartheta_{1}+\vartheta_{2}\left(I_{m}+\vartheta_{2}^{*} \vartheta_{2}\right) \\
I_{m}+\vartheta_{2}^{*} \vartheta_{2} \\
-\vartheta_{2}
\end{array}\right], \\
& \hat{J}=\left[\begin{array}{ccc}
0 & 0 & I_{n} \\
0 & I_{m} & 0 \\
I_{n} & 0 & 0
\end{array}\right], \quad C=\left[\vartheta_{2}^{*} I_{m}-\vartheta_{2}^{*} \vartheta_{2}-\vartheta_{1}^{*}+\left(I_{m}-\vartheta_{2}^{*} \vartheta_{2}\right) \vartheta_{2}^{*}\right] .
\end{aligned}
$$

Moreover, for any sufficiently large values of $\Im \sqrt{z}$ the matrix $\phi(\sqrt{z})$, such that (4.6) holds, is unique.

According to Theorem 4.1 inequality (2.22) holds for

$$
M(z)=-\phi(\sqrt{z})^{-1}
$$

and sufficiently large values of $\Im \sqrt{z}$. From inequality (2.61) in [14] follows also that matrices $M(z)$ satisfying (2.22) for sufficiently large values of $\Im \sqrt{z}$ are unique.

By Proposition 2.3 in [14] we have

$$
\sup _{0 \leq x<\infty}\|u(x)\|<\infty .
$$

Thus, there is a unique Weyl function $M(z)$ of system (2.21) and this Weyl function satisfies (2.22). Therefore, equality (4.10) defines the Weyl function $M(z)$ for sufficiently large values of $\Im \sqrt{z}$. Note also that the Weyl function $M$ and the matrix function $\phi(\sqrt{z})^{-1}$ are meromorphic.

Corollary 4.2. The Weyl function $M$ of system (2.21), where $u$ has the form (4.2), is given by formulas (4.7)-(4.10) for all $z \in \mathbb{C}_{+}$excluding a finite number of points.

Relation (4.2) can be rewritten as

$$
u=2\left(\Omega_{22}^{2}+\Omega_{12}+\Omega_{21}\right) ; \quad \Omega_{k j}:=\Lambda_{k}^{*} S^{-1} \Lambda_{j}, \quad k, j=1,2 .
$$

The derivatives of $u$ are calculated in [14] using (4.2)-(4.5). In particular, from the expressions (5.16) and (5.17) in [14] for the derivatives of $\Omega_{k j}$ one can get

$$
u_{x}=2\left(\Lambda_{2}^{*} \alpha^{*} S^{-1} \Lambda_{2}+\Lambda_{2}^{*} S^{-1} \alpha \Lambda_{2}-2 \Omega_{11}-\Omega_{12} \Omega_{22}-\Omega_{22} \Omega_{21}\right)-u \Omega_{22}-\Omega_{22} u .
$$

Formula (5.31) in [14] has the form

$$
\begin{aligned}
3 u^{2}-\frac{\partial^{2} u}{\partial x^{2}}= & 8\left(\Omega_{21} \Omega_{12}+\Lambda_{2}^{*} S^{-1} \alpha \Lambda_{2} \Omega_{22}+\Omega_{22} \Lambda_{2}^{*} \alpha^{*} S^{-1} \Lambda_{2}\right. \\
& \left.+\Lambda_{2}^{*} S^{-1} \alpha \Lambda_{1}+\Lambda_{1}^{*} \alpha^{*} S^{-1} \Lambda_{2}\right) .
\end{aligned}
$$

Formula (5.37) in [14] after some cancellations takes the form

$$
\begin{aligned}
& \frac{\partial}{\partial x}\left(3 u^{2}-\frac{\partial^{2} u}{\partial x^{2}}\right)=8\left\{\Lambda_{2}^{*}\left(\alpha^{*}\right)^{2} S^{-1} \Lambda_{2}-\Lambda_{1}^{*} \alpha^{*} S^{-1} \Lambda_{1}-\Lambda_{1}^{*} \alpha^{*} S^{-1} \Lambda_{2} \Omega_{22}\right. \\
& +\Lambda_{2}^{*} S^{-1} \alpha^{2} \Lambda_{2}-\Lambda_{1}^{*} S^{-1} \alpha \Lambda_{1}-\Omega_{22} \Lambda_{2}^{*} S^{-1} \alpha \Lambda_{1}-\Lambda_{2}^{*} S^{-1} \alpha \Lambda_{2}\left(\Omega_{22}^{2}+\Omega_{21}\right) \\
& -\left(\Lambda_{2}^{*} S^{-1} \alpha \Lambda_{1}+\Lambda_{1}^{*} S^{-1} \alpha \Lambda_{2}+\Omega_{22} \Lambda_{2}^{*} S^{-1} \alpha \Lambda_{2}\right) \Omega_{22}-\left(\Omega_{22}^{2}+\Omega_{12}\right) \Lambda_{2}^{*} \alpha^{*} S^{-1} \Lambda_{2} \\
& -\Omega_{22}\left(\Lambda_{2}^{*} \alpha^{*} S^{-1} \Lambda_{1}+\Lambda_{1}^{*} \alpha^{*} S^{-1} \Lambda_{2}+\Lambda_{2}^{*} \alpha^{*} S^{-1} \Lambda_{2} \Omega_{22}\right)-\left(\Omega_{22} \Omega_{21}+\Omega_{11}\right) \Omega_{12} \\
& \left.-\Omega_{21}\left(\Omega_{12} \Omega_{22}+\Omega_{11}\right)\right\} .
\end{aligned}
$$

Our next proposition follows from (4.12)-(4.15). 
Proposition 4.3. Let

$$
\alpha=\alpha^{*}, \quad \vartheta_{1}^{*} \alpha \vartheta_{1}=0, \quad \vartheta_{2}=0 .
$$

Then the triple $\left\{\alpha, \vartheta_{1}, \vartheta_{2}\right\}$ is admissible and

$$
u(0)=u_{x x}(0)=u_{x x x}(0)=0, \quad u_{x}(0)=-4 \vartheta_{1}^{*} \vartheta_{1} .
$$

Proof. As $\alpha=\alpha^{*}$ and $\vartheta_{2}=0$ the identity (4.1) holds, that is, the triple $\left\{\alpha, \vartheta_{1}, \vartheta_{2}\right\}$ is admissible. According to (4.4) and (4.16) we have $\Lambda_{2}(0)=\vartheta_{2}=0$. As $\Lambda_{2}(0)=0$ we have also $\Omega_{21}(0)=\Omega_{12}(0)=$ $\Omega_{22}(0)=0$, and so formula (4.12) implies $u(0)=0$. Taking into account that

$$
u(0)=0, \quad \Lambda_{2}(0)=0, \quad \Omega_{21}(0)=\Omega_{12}(0)=\Omega_{22}(0)=0, \quad S(0)=I_{n},
$$

we derive from formulas (4.4) and (4.13) the equality $u_{x}(0)=-4 \vartheta_{1}^{*} \vartheta_{1}$. Moreover, formulas (4.14) and (4.18) yield $u_{x x}(0)=0$. By (4.15) and (4.18) we have

$$
u_{x x x}(0)=8 \Lambda_{1}(0)^{*}\left(\alpha^{*} S(0)^{-1}+S(0)^{-1} \alpha\right) \Lambda_{1}(0)=8 \vartheta_{1}^{*}\left(\alpha+\alpha^{*}\right) \vartheta_{1} .
$$

Finally, in view of (4.16) and (4.19) we get $u_{x x x}(0)=0$.

The first three equalities in (4.17) mean that the initial condition $u(x, 0)=u(x)$ for $\mathrm{KdV}$ complies with the boundary conditions $u(0, t)=u_{x x}(0, t)=0$.

Example 4.4. Consider the case

$$
\alpha=0, \quad \vartheta_{2}=0 .
$$

It is immediate that (4.16) holds, that is, the conditions of Proposition 4.3 are fulfilled. It easily follows from (4.4), (4.5), and (4.20) that

$$
e^{x \beta}=I_{2 n}+x \beta, \quad \Lambda_{1} \equiv \vartheta_{1}, \quad \Lambda_{2}(x)=-x \vartheta_{1}, \quad S(x)=I_{n}+\frac{1}{3} x^{3} \vartheta_{1} \vartheta_{1}^{*} .
$$

Taking into account (4.21), we derive from (4.2) that

$$
u(x)=2 x^{4} \vartheta_{1}^{*}\left(I_{n}+\frac{1}{3} x^{3} c\right)^{-1} c\left(I_{n}+\frac{1}{3} x^{3} c\right)^{-1} \vartheta_{1}-4 x \vartheta_{1}^{*}\left(I_{n}+\frac{1}{3} x^{3} c\right)^{-1} \vartheta_{1},
$$

where $c:=\vartheta_{1} \vartheta_{1}^{*}$. The Weyl function of system (2.21), where $u$ is given by (4.22), is constructed using (4.7)-(4.10) and (4.20). First note that

$$
\left(z I_{2 n+m}-A\right)^{-1}=\left[\begin{array}{llr}
z^{-1} I_{n} & 0 & 0 \\
z^{-2} \vartheta_{1}^{*} & z^{-1} I_{m} & 0 \\
z^{-3} c & z^{-2} \vartheta_{1} & z^{-1} I_{n}
\end{array}\right] .
$$

Hence, we obtain

$$
\begin{aligned}
& \varphi_{1}(z)^{-1}=I_{m}+z^{-1} I_{m}+2 z^{-2} \widehat{c}+z^{-3} \widehat{c}^{2}, \quad \widehat{c}:=\vartheta_{1}^{*} \vartheta_{1}, \\
& \varphi_{2}(z)=-I_{m}+z^{-1} I_{m}-z^{-3} \widehat{c}^{2} .
\end{aligned}
$$

Substitute (4.24) and (4.25) into (4.7), and substitute the result into (4.10) to get

$$
M(z)=\sqrt{z}\left(z^{3} I_{m}+\left(z I_{m}+\widehat{c}\right)^{2}\right)\left(\sqrt{z}\left(z^{3} I_{m}-z^{2} I_{m}+\widehat{c}^{2}\right)-2 i z^{3} I_{m}\right)^{-1} .
$$

We have $\widehat{c}=\vartheta_{1}^{*} \vartheta_{1} \geq 0$. Assume for simplicity $\widehat{c}>0$. Then, according to (4.26) the low energy asymptotics of $M$ is given by the formula

$$
M(z)=I_{m}+2 z \widehat{c}^{-1}+O\left(z^{2}\right) \quad(z \rightarrow 0)
$$


Though equalities (4.17) for $u(x, 0)=u(x)$ comply with the boundary conditions $u(0, t)=u_{x x}(0, t)=0$ the following non-existence proposition is true.

Proposition 4.5. There is no solution $u$ of the $K d V$ equation with a negative dispersion term in the quarter-plane $x \geq 0, t \geq 0$, such that $u(x, t)$ satisfies conditions of Proposition 3.2, where the initial condition in (3.11) is determined by the admissible triple $\left\{0, \vartheta_{1}, 0\right\}\left(\widehat{c}=\vartheta_{1}^{*} \vartheta_{1}>0\right)$, namely, $u(x, 0)$ has the form:

$$
u(x, 0)=2 x^{4} \vartheta_{1}^{*}\left(I_{n}+\frac{1}{3} x^{3} c\right)^{-1} c\left(I_{n}+\frac{1}{3} x^{3} c\right)^{-1} \vartheta_{1}-4 x \vartheta_{1}^{*}\left(I_{n}+\frac{1}{3} x^{3} c\right)^{-1} \vartheta_{1} .
$$

Proof. We prove this proposition by contradiction. Suppose that $u(x, t)$ described in the proposition exists. Then $M(0, z)=M(z)$, where $M$ is given by (4.26). Hence, by Proposition 3.2 the Weyl function of system (3.19) is given by the formula

$$
M_{D}\left(-4 z^{\frac{3}{2}}\right)=\frac{1}{\sqrt{z}}\left(I_{m}+M(z)\right)\left(I_{m}-M(z)\right)^{-1}
$$

for sufficiently large values of $\Im \sqrt{z}$, where $z$ belongs to the sector $\frac{2}{3} \pi<\arg (z)<\pi$. Recall that as a Wel function $M_{D}(\zeta)$ is a Herglotz function $\left(\zeta \in \mathbb{C}_{+}\right)$and that $M(z)$ is meromorphic in $\mathbb{C}$. Note that

$$
\frac{2}{3} \pi<\arg (z)<\frac{4}{3} \pi
$$

implies $-4 z^{\frac{3}{2}} \in \mathbb{C}_{+}$. Therefore (4.29) holds in the sector (4.30). The asymptotics (4.27) holds in $\mathbb{C}$ and, in particular, in the sector (4.30) too. Moreover, according to (4.27) and (4.29) the low energy asymptotics of $M_{D}$ has the form

$$
M_{D}\left(-4 z^{\frac{3}{2}}\right)=-z^{-\frac{3}{2}}\left(I_{m}+O(z)\right) \widehat{c}, \quad z \rightarrow 0,
$$

which contradicts the Herglotz property of $M_{D}$.

Put

$$
\begin{aligned}
& \Lambda(x, t)=\left[\begin{array}{l}
\Lambda_{1}(x, t) \\
\Lambda_{2}(x, t)
\end{array}\right]=e^{x \beta+4 t \beta^{3}}\left[\begin{array}{l}
\vartheta_{1} \\
\vartheta_{2}
\end{array}\right], \quad \beta=\left[\begin{array}{lr}
0 & \alpha \\
-I_{n} & 0
\end{array}\right], \\
& S(x, t)=I_{n}+P_{1}\left[\begin{array}{ll}
0 & e^{x \beta+4 t \beta^{3}}
\end{array}\right] e^{x \omega+4 t \omega^{3}}\left[\begin{array}{c}
P_{1}^{*} \\
0
\end{array}\right], \quad \omega=\left[\begin{array}{ll}
\beta^{*} & 0 \\
b & -\beta
\end{array}\right],
\end{aligned}
$$

where $\left\{\alpha, \vartheta_{1}, \vartheta_{2}\right\}$ is an admissible triple, $P_{1}=\left[\begin{array}{ll}0 & I_{n}\end{array}\right], b=\left[\begin{array}{l}\vartheta_{1} \\ \vartheta_{2}\end{array}\right]\left[\begin{array}{ll}\vartheta_{1}^{*} & \vartheta_{2}^{*}\end{array}\right]$. Then, according to Theorem 0.5 in [14] the matrix function $u(x, t)$, given by (4.12) in the points of invertibility of $S$, satisfies KdV (1.1). Notice that $\Lambda(x, 0)$ and $S(x, 0)$ defined above coincide with $\Lambda(x)$ and $S(x)$ in (4.4) and (4.5), respectively. Moreover, according to Chapter 5 in [14] equalities (4.13)-(4.15) hold for each $t$. Finally, from (5.6) and (5.9) in [14] we have

$$
S_{x}=\Lambda_{2} \Lambda_{2}^{*}, \quad S_{t}=-4\left(\alpha \Lambda_{2} \Lambda_{2}^{*}+\Lambda_{2} \Lambda_{2}^{*} \alpha^{*}+\Lambda_{1} \Lambda_{1}^{*}\right) .
$$

(We changed $\Lambda(x, t)$ into $\Lambda(x,-t), S(x, t)$ into $S(x,-t)$, and $u(x, t)$ into $u(x,-t)$ in the expressions in [14] to obtain $\mathrm{KdV}$ solutions with a negative dispersion term.)

Example 4.6. Blow-up solutions.

Consider again the case (4.20) of the triple $\left\{0, \vartheta_{1}, 0\right\}$, where $\vartheta_{1} \neq 0$. By $(4.20)$ we see that $\beta^{2}=\beta^{3}=0$. As $\beta^{3}=0$ formulas (4.20) and (4.32) imply

$$
\Lambda_{1}(x, t) \equiv \vartheta_{1}, \quad \Lambda_{2}(x, t)=-x \vartheta_{1}
$$


(compare with (4.21)). In particular, we get $\Lambda_{2}(0, t) \equiv 0$. Hence, in view of $(4.12)$ and (4.14) we derive

$$
u(0, t)=u_{x x}(0, t)=0
$$

in the points of invertibility of $S(0, t)$. It follows from (4.34), (4.35), and equality $\alpha=0$ that

$$
S(x, t)=I_{n}+\left(\frac{1}{3} x^{3}-4 t\right) c, \quad c=\vartheta_{1} \vartheta_{1}^{*} .
$$

Substitute (4.35) and (4.37) into (4.12) to get

$$
\begin{aligned}
u(x, t)= & 2 x^{4} \vartheta_{1}^{*}\left(I_{n}+\left(\frac{1}{3} x^{3}-4 t\right) c\right)^{-1} c\left(I_{n}+\left(\frac{1}{3} x^{3}-4 t\right) c\right)^{-1} \vartheta_{1} \\
& -4 x \vartheta_{1}^{*}\left(I_{n}+\left(\frac{1}{3} x^{3}-4 t\right) c\right)^{-1} \vartheta_{1} .
\end{aligned}
$$

The blow-up should occur when $\operatorname{det} S(x, t)$ turns to zero. In the simplest case $n=1$ formula (4.38) takes the form

$$
u(x, t)=\frac{\frac{2}{3} c x^{4}+16 c x t-4 x}{\left(1+\frac{1}{3} c x^{3}-4 c t\right)^{2}} \vartheta_{1}^{*} \vartheta_{1},
$$

and for $t \geq \frac{1}{4 c}$ we have singularity at $x=(3(4 c t-1) / c)^{\frac{1}{3}}$.

Our next proposition deals with the case, where $\operatorname{det} \alpha \neq 0$ and low energy asymptotics of $M$ is different from the asymptotics in (4.27) but the global solutions $u$ again do not exist.

Proposition 4.7. There is no solution $u$ of the KdV equation with a negative dispersion term in the quarter-plane $x \geq 0, t \geq 0$, such that $u(x, t)$ satisfies conditions of Proposition 3.2, where $u(x, 0)$ is determined by the triple $\left\{\alpha, \vartheta_{1}, 0\right\}$, which satisfies relations

$$
\alpha=\alpha^{*}, \quad \vartheta_{1}^{*} \alpha \vartheta_{1}=0, \quad \operatorname{det} \alpha \neq 0, \quad \operatorname{det}\left(I_{m} \pm \vartheta_{1}^{*} \alpha^{-1} \vartheta_{1}\right) \neq 0, \quad \vartheta_{1}^{*} \alpha^{-1} \vartheta_{1} \not \leq 0 .
$$

Proof. As $\vartheta_{2}=0, \alpha=\alpha^{*}$, and $\vartheta_{1}^{*} \alpha \vartheta_{1}=0$ the triple is admissible and equalities (4.17) hold, that is, the initial condition complies with the boundary conditions $u(0, t)=u_{x x}(0, t)=0$. By (4.9) we have

$$
\begin{aligned}
& \left(z I_{2 n+m}-A\right)^{-1}=\left[\begin{array}{lcr}
\left(z I_{n}-\alpha\right)^{-1} & 0 & 0 \\
z^{-1} \vartheta_{1}^{*}\left(z I_{n}-\alpha\right)^{-1} & z^{-1} I_{m} & 0 \\
z^{-1} c_{1}(z) c\left(z I_{n}-\alpha\right)^{-1} & z^{-1} c_{1}(z) \vartheta_{1} & c_{1}(z)
\end{array}\right], \\
& B^{*}=\left[\begin{array}{lll}
\vartheta_{1}^{*} I_{m} & 0
\end{array}\right], \quad C=\left[\begin{array}{ll}
0 I_{m}-\vartheta_{1}^{*}
\end{array}\right],
\end{aligned}
$$

where

$$
c_{1}(z)=\left(z I_{n}-\alpha\right)^{-1}, \quad c=\vartheta_{1} \vartheta_{1}^{*} .
$$

According to (4.8), (4.41), and (4.42) we have

$$
\begin{aligned}
& \varphi_{1}(z)^{-1}=I_{m}+z^{-1}\left(I_{m}+\vartheta_{1}^{*}\left(z I_{n}-\alpha\right)^{-1} \vartheta_{1}\right)^{2}, \\
& \varphi_{2}(z)=-I_{m}+z^{-1}\left(I_{m}-\left(\vartheta_{1}^{*}\left(z I_{n}-\alpha\right)^{-1} \vartheta_{1}\right)^{2}\right) .
\end{aligned}
$$

By (4.7), (4.10), (4.43), and (4.44) the low energy asymptotics of $M(0, z)$ has the form

$$
\begin{aligned}
M(0, z)= & -\left(I_{m}+\vartheta_{1}^{*} \alpha^{-1} \vartheta_{1}\right)^{-1}\left(I_{m}-\vartheta_{1}^{*} \alpha^{-1} \vartheta_{1}-2 i \sqrt{z}\left(I_{m}+\vartheta_{1}^{*} \alpha^{-1} \vartheta_{1}\right)^{-1}\right) \\
& +O(z), \quad z \rightarrow 0 .
\end{aligned}
$$


Finally, in a way similar to the corresponding part of the proof of Proposition 4.5 we assume that $u(x, t)$ satisfying conditions of Proposition 3.2 exists and get

$$
M_{D}\left(-4 z^{\frac{3}{2}}\right)=\frac{1}{\sqrt{z}} \vartheta_{1}^{*} \alpha^{-1} \vartheta_{1}+O(1), \quad z \rightarrow 0
$$

in the sector (4.30). In view of the last relation in (4.40) this means that $M_{D}$ does not belong to Herglotz class and we come to a contradiction.

Acknowledgements. This work was supported by the Austrian Science Fund (FWF) under Grant no. Y330.

The author is very grateful to F. Gesztesy for his help and interest in the topic.

The author is grateful also to the referee for the careful reading and useful remarks.

\section{References}

[1] J.L. Bona, A.S. Fokas. Initial-boundary-value problems for linear and integrable nonlinear dispersive equations. Nonlinearity, 21 (2008), T195-T203.

[2] J.L. Bona, W.G. Pritchard, L.R. Scott. An evaluation of a model equation for water waves. Philos. Trans. R. Soc. Lond., A 302 (1981), 458-510.

[3] J. Bona, R. Winther. The Korteweg-de Vries equation, posed in a quarter-plane. SIAM J. Math. Anal., 14 (1983), 1056-1106.

[4] R. Carroll, Q. Bu. Solution of the forced nonlinear Schrödinger (NLS) equation using PDE techniques. Appl. Anal., 41 (1991), 33-51.

[5] C.K. Chu, L.W. Xiang, Y. Baransky. Solitary waves induced by boundary motion. Commun. Pure Appl. Math., 36 (1983), 495-504.

[6] S. Clark, F. Gesztesy. Weyl-Titchmarsh M-function asymptotics for matrix-valued Schrödinger operators. Proc. Lond. Math. Soc., III. Ser., 82 (2001), 701-724.

[7] S. Clark, F. Gesztesy, M. Zinchenko. Weyl-Titchmarsh theory and Borg-Marchenko-type uniqueness results for CMV operators with matrix-valued Verblunsky coefficients. Oper. Matrices, 1 (2007), 535-592.

[8] A.S. Fokas. Integrable nonlinear evolution equations on the half-line. Comm. Math. Phys., 230 (2002), 1-39.

[9] A.S. Fokas. A unified approach to boundary value problems. CBMS-NSF Regional Conference Ser. in Appl. Math. vol. 78. SIAM, Philadelphia, 2008.

[10] A.S. Fokas, J. Lenells. Explicit soliton asymptotics for the Korteweg-de Vries equation on the half-line. Nonlinearity, 23 (2010), 937-976.

[11] G. Freiling, V. Yurko. Inverse Sturm-Liouville Problems and Their Applications. Nova Science Publishers, Huntington, N.Y., 2001.

[12] F. Gesztesy, B. Simon. On local Borg-Marchenko uniqueness results. Commun. Math. Phys., 211 (2000), $273-287$.

[13] F. Gesztesy, B. Simon. A new approach to inverse spectral theory. II. General real potentials and the connection to the spectral measure. Ann. of Math. (2), 152 (2000), 593-643.

[14] I. Gohberg, M.A. Kaashoek, A.L. Sakhnovich. Sturm-Liouville systems with rational Weyl functions: explicit formulas and applications. Integr. Equ. Oper. Theory, 30 (1998), 338-377.

[15] M. Kac, P. van Moerbeke. A complete solution of the periodic Toda problem. Proc. Natl. Acad. Sci. USA, 72 (1975), 2879-2880.

[16] D.J. Kaup, H. Steudel. Recent results on second harmonic generation. Contemp. Math., 326 (2003), 33-48.

[17] A. Kostenko, A. Sakhnovich, G. Teschl. Weyl-Titchmarsh theory for Schrödinger operators with strongly singular potentials. Int. Math. Res. Not. 2011, Art. ID rnr065, 49pp.

[18] P.C. Sabatier. Elbow scattering and inverse scattering applications to LKdV and KdV. J. Math. Phys., 41 (2000), 414-436.

[19] P.C. Sabatier. Lax equations scattering and KdV. J. Math. Phys., 44 (2003), 3216-3225.

[20] P.C. Sabatier. Generalized inverse scattering transform applied to linear partial differential equations. Inverse Probl., 22 (2006), 209-228.

[21] A.L. Sakhnovich. Dirac type and canonical systems: spectral and Weyl-Titchmarsh fuctions, direct and inverse problems. Inverse Probl., 18 (2002), 331-348.

[22] A.L. Sakhnovich. Second harmonic generation: Goursat problem on the semi-strip, Weyl functions and explicit solutions. Inverse Probl., 21 (2005), 703-716.

[23] A.L. Sakhnovich. On the compatibility condition for linear systems and a factorization formula for wave functions. J. Differ. Equations, 252 (2012), 3658-3667.

[24] A.L. Sakhnovich. Sine-Gordon theory in a semi-strip. Nonlinear Analysis, 75 (2012), 964-974.

[25] L.A. Sakhnovich. Nonlinear equations and inverse problems on the semi-axis (Russian). Preprint 87.30. Mathematical Institute, Kiev, 1987.

[26] L.A. Sakhnovich. Evolution of spectral data, and nonlinear equations. Ukrain. Math. J., 40 (1988), $459-461$. 
[27] L.A. Sakhnovich. Spectral Theory of Canonical Differential Systems. Method of Operator Identities. Operator Theory Adv. Appl. Ser. vol. 107. Birkhäuser, Basel, 1999.

[28] B.A. Ton. Initial boundary value problems for the Korteweg-de Vries equation. J. Differ. Equations 25 (1977), 288-309.

[29] P.A. Treharne, A.S. Fokas. The generalized Dirichlet to Neumann map for the KdV equation on the half-line. J. Nonlinear Sci., 18 (2008), 191-217. 